\title{
Utility investigation of artificial time delay in displacement-noise-free interferometers
}

\author{
Kentaro Somiya and Yanbei Chen \\ Max-Planck Institut für Gravitationsphysik, Am Mhlenberg 1, 14476 Potsdam, Germany \\ Keisuke Goda \\ LIGO Laboratory, Massachusetts Institute of Technology, Cambridge, Massachusetts 02139, USA \\ Eugeniy E. Mikhailov \\ Department of Physics, College of William Mary, Williamsburg, Virginia 23187, USA
}

(Received 27 March 2007; published 10 July 2007)

\begin{abstract}
Laser interferometer gravitational wave detectors are usually limited by displacement noise in their lower frequency band. Recently, theoretical proposals have been put forward to construct schemes of interferometry that are insusceptible to displacement noise as well as classical laser noise. These so-called displacement-noise-free interferometry (DFI) schemes take advantage of the difference between gravitational waves and displacement noise in their effects on light propagation. However, since this difference diminishes in lower frequencies (i.e., $\Omega<c / \mathcal{L}_{D}$, with $\mathcal{L}_{D}$ the size of the detector), shot-noise-limited sensitivity of DFI schemes deteriorates dramatically in these frequencies-exactly the regime in which they are supposed to be superior, thereby limiting their applicability. In this paper, we explore the obvious possibility of increasing the effective size of the detector in the time domain, by introducing artificial time delays $\left(\mathcal{T}_{D} \gg \mathcal{L}_{D} / c\right)$ into the interferometry scheme, with the hope of improving low-frequency sensitivity. We found that sensitivity can only be improved by schemes in which fluctuations in the artificial time delays are not canceled.
\end{abstract}

DOI: 10.1103/PhysRevD.76.022002

PACS numbers: 04.80.Nn, 07.60.Ly, 42.30.Lr, 95.55.Ym

\section{INTRODUCTION}

Gravitational waves (GWs) are "ripples" of space-time curvature caused by accelerated motion of mass-energy, according to the prediction of general relativity. Detecting GWs from the distant universe will provide a new probe to the strong-field regime of general relativity (from which theses waves often originate), as well as a new window to high-energy astrophysical processes.

A broad category of GW detectors, namely, laser interferometric GW detectors (interferometers for short), detect GWs by sending light between various test masses and measuring changes in light travel times (or phase shifts) due to incoming GWs [1-5]. Laser interferometers are susceptible, at least at first sight, to two types of noise: (i) those due to uncertainties in timing, or timing noise, e.g., classical and quantum fluctuations in the laser light, and (ii) those due to uncertainties in the motions of test masses, or displacement noise, e.g., seismic noise in ground-based interferometers and acceleration noise in space-based interferometers.

In laser interferometers, classical timing noise (laser frequency noise) can be suppressed dramatically. In these devices, one laser beam is first split into two; each of these beams is then sent to travel a different path, picking up a different signal from the other. The two different paths are designed to meet again after having traveled the same distance, with the two beams recombined with phase subtraction. At this point, the two beams share the same classical phase noise. The subtraction therefore provides a classical laser-noise-free differential signal. The level of timing-noise suppression depends on, e.g., the accuracy at which the lengths of the paths are balanced, and the balancing of optical losses between these two paths. It is worth noting that this method cannot cancel quantum noise, because independent vacuum fluctuation is introduced when the beam is first split, and the two beams do not share the same quantum laser noise. In this paper, we shall use shot noise to denote quantum timing noise. Shot noise can be lowered by increasing laser power.

Recently, Kawamura and Chen showed that it is possible to remove both classical timing noise and (classical and quantum) displacement noise by combining light travel times among an array of test masses [6-9]. Such schemes are called displacement-noise-free interferometry, or DFI. DFI is enabled by the theoretical fact that GWs with nonzero frequencies affect light propagation differently from noisy motions of mirrors (although this difference is rather small, when the spatial size of the detector, $\mathcal{L}_{D}$, is small compared to the wavelength of the $\mathrm{GW}, \lambda_{\mathrm{GW}}$ ) $[6,10]$. In practice, DFI must be realized by allowing multiple light beams to reflect off each mirror, at different incident angles (in 2-D configurations) and/or from different planes (in 3D configurations), such that these multiple beams sense the mirror motion redundantly, while sensing the GW field differently $[8,11]$.

A shortcoming of DFI arises from the fact that when $2 \pi \mathcal{L}_{D} \lesssim \lambda_{\mathrm{GW}}$ (i.e., $\Omega_{\mathrm{GW}}<\Omega_{\text {peak }} \equiv c / \mathcal{L}_{D}$ ), the effect of GW can be well approximated by a tidal field, which induces relative mirror motions, without affecting propagation of light. As a consequence, DFI schemes become 
significantly less sensitive to GWs at lower frequencies. To be more precise, we have a two-part power law:

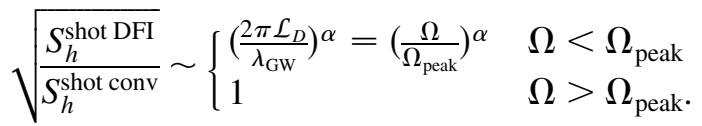

Here $S_{h}^{\text {shot DFI }}$ and $S_{h}^{\text {shot conv }}$ are the shot-noise spectral densities of DFI and conventional configurations with comparable spatial sizes, with $\alpha=3$ for 2-D DFI configurations, and $\alpha=2$ for 3-D DFI configurations. This strongly limits the applicability of DFI schemes. For ground-based detectors, we usually operate at frequencies much lower than $\Omega_{\text {peak }}$. For space-based detectors like LISA, although $\Omega_{\text {peak }}$ is within our detection band, it is still above the frequency at which displacement noise (acceleration noise) dominates; since acceleration noise is $\sim f^{-2}$ at low frequencies, the shot-noise-limited DFI sensitivity still cannot surpass the combined sensitivity of conventional detectors.

In order to extend the applicability of existing DFI schemes, one might consider (i) increasing the effective spatial size of a DFI scheme by using Fabry-Perot cavities or optical delay lines, or (ii) increasing the effective temporal size of a DFI scheme by using a time-delay device. Approach (i) has been explored by Nishizawa et al. [12]; the result does not go beyond the expected improvement that is achievable by applying power and signal recycling to the existing DFI configuration in Ref. [8].

In this paper, we explore approach (ii), with the hope that a sensitivity with $\alpha<2$ [cf. Eq. (1)] might be obtained. In DFI configurations, timing signals are subtracted from each other in order to cancel displacement and timing noise. A GW signal can partially survive these subtractions, because these signals sense the GW field in a distributed way, over spatial and temporal extent of $\mathcal{L}_{D}$ (if we set $c=1)$. However, as argued before, when $2 \pi \mathcal{L}_{D}<$ $\lambda_{\mathrm{GW}}$, these subtractions suppress $\mathrm{GW}$ sensitivity by $\left(2 \pi \mathcal{L}_{D} / \lambda_{\mathrm{GW}}\right)^{\alpha}$. Now, if we introduce long time delays $\sim \mathcal{T}_{D} \gg \mathcal{L}_{D}$ into our scheme, there will be two types of subtractions, those with $\mathcal{L}_{D}$ in spatial separation, and those with $\mathcal{T}_{D}$ in temporal separation. We would expect (i) cancellation at a lower order if $\Omega_{\text {peak }}>\Omega>\Omega_{\text {delay }} \equiv$ $1 / \mathcal{T}_{D}$, and (ii) enhancement of sensitivity by some power of $\sim \mathcal{T}_{D} / \mathcal{L}_{D} \sim \Omega_{\text {peak }} / \Omega_{\text {delay }}$, if $\Omega<\Omega_{\text {delay }}$. Taking 3-D configurations as an example, we might expect the following, three-part power law:

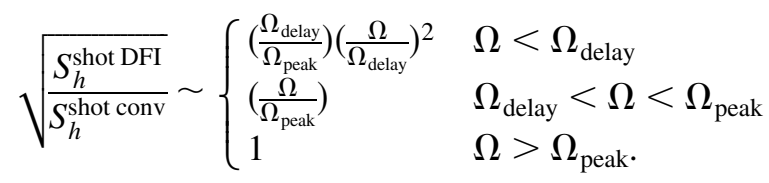

In addition to testing whether such a new power law appears in the shot-noise-limited sensitivity, an important question is whether fluctuations in the artificial time delays introduce noise, and whether they can be canceled without affecting GW sensitivity.
This paper is organized as follows. In Sec. II, we briefly review the concept of DFI without time delay, using spacetime diagrams. In Sec. III, we introduce the doubleoctahedron geometry on which interferometry schemes discussed in this paper will be based. In Sec. IV, we show a few examples that have been introduced in Ref. [11] as the DFI configurations with time delay, in which delay noise is canceled or partially canceled; these configurations either fail to cancel displacement noise or follow the two-part power law (1) with $\alpha=2$. In Sec. V, we theoretically demonstrate the general incompatibility between sensitivity improvement and delay-noise cancellation; we also provide an example scheme in which sensitivity improvement is indeed achieved, at the price of being susceptible to delay noise. In Sec. VI, we summarize our main conclusions. In the appendix, we discuss delay noise in the case with a time-delay device based on electromagnetically-induced-transparency (EIT) [13].

\section{DFI WITHOUT TIME DELAY}

In Fig. 1, we use a 2-D $(1+1)$ space-time diagram to make a comparison between conventional Michelson interferometry and DFI. In both situations, we have light pulses departing from $X_{1}$ toward two opposite directions, arriving at $Y_{1}$ and $Y_{2}$, immediately reflected back, and subsequently arriving at the test mass from which they originated, around $X_{2}$. Note that $Y_{1}$ and $Y_{2}$ refer to two different objects, while $X_{1}$ and $X_{2}$ refer to the same object at different times. We use the transverse-traceless (TT) gauge (see Ref. [6] for details), in which ideal test masses follow geodesics and stay in constant spatial coordinates. Nongeodesic test-mass motions are then the displacement noise in the experiment. Up to linear order in GW amplitude and displacement noise, the proper time of the test masses, which ideal clocks are supposed to indicate, agree with the coordinate time. In absence of $\mathrm{GW}$, displacement noise and timing noise, $Y_{1}$ and $Y_{2}$, are equidistant from $X$, light pulses arrive at $Y_{1,2}$ simultaneously, and return to $X_{2}$ simultaneously — as indicated by the local clocks. In the

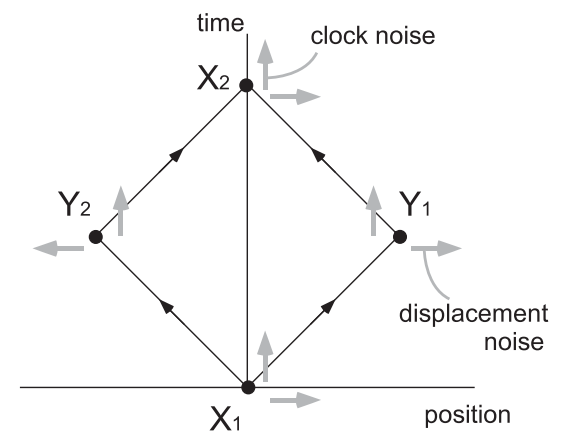

FIG. 1. 2-D space-time diagram comparing conventional Michelson interferometry (which cancels clock noise, but not displacement noise) with DFI in Ref. [6] (cancels displacement noise, but not clock noise). 
diagram, we use vertical arrows to represent timing noise, and horizontal arrows to represent displacement noise. When GW and the two types of noise are present, the time the pulses take to travel between $X_{i}$ and $Y_{j}, i, j=$ 1,2 , as indicated by the clocks, will differ from the ideal situation, we use $\tau_{X_{i} Y_{j}}$, to indicate these differences.

In conventional Michelson interferometry, light travel times along the $X_{1} Y_{1} X_{2}$ path and the $X_{1} Y_{2} X_{2}$ path are compared. In other words,

$$
\tau_{\mathrm{MI}}=\left(\tau_{X_{1} Y_{1}}+\tau_{Y_{1} X_{2}}\right)-\left(\tau_{X_{1} Y_{2}}+\tau_{Y_{2} X_{2}}\right)
$$

is measured. In this case, clock noise at $X_{1,2}$ does not appear since the time delays $\tau_{X_{j} Y_{1}}$ and $\tau_{X_{j} Y_{2}}$ that include the same clock noise are subtracted at the output. On the other hand, displacement noise at $Y_{j}$ does not cancel since $\tau_{X_{1} Y_{j}}$ and $\tau_{X_{2} Y_{j}}$ are added. Clock noise at $Y_{j}$ does not appear since the time delays are added but they sense clock noise with different signs, which can be seen by the direction of the gray arrow in terms of the black arrow in the figure. Displacement noise at $X_{j}$ appears since the phase shifts are subtracted but with different signs as well.

In the Kawamura-Chen 1-D DFI configuration (Ref. [6]), the following timing combination is measured:

$$
\tau_{\mathrm{DFI}}=\left(\tau_{X_{1} Y_{1}}+\tau_{X_{1} Y_{2}}\right)-\left(\tau_{Y_{1} X_{2}}+\tau_{Y_{2} X_{2}}\right) .
$$

In this combination, displacement noise at $X_{j}$ or $Y_{j}$ does not appear. However, timing noise does appear. It is interesting to note that, in the space-time diagram, the DFI configuration is $90^{\circ}$ rotated from the Michelson configuration. This DFI configuration has shot-noise-limited sensitivity with $\alpha=1$.

As shown in Ref. [7], in order to cancel both displacement and timing noise without canceling GW completely, we must use configurations with 2 or 3 spatial dimensions. One part of such a scheme is shown in a $(2+1)$ space-time diagram in Fig. 2. In this scheme, light pulses are sent from $A$ and $B$ toward $C$, getting reflected, and then reaching $B^{\prime}$ and $A^{\prime}$, respectively. The timing combination is

$$
\left(\tau_{A C}+\tau_{C B^{\prime}}\right)-\left(\tau_{B C}+\tau_{C A^{\prime}}\right) .
$$

Note that $A$ and $B$ are equidistant from $C$; as a consequence, in absence of GW and noise, the pulses they send arrive at $C$ simultaneously. In this situation, we have three noise channels at $C$ : timing noise is canceled within each of the brackets; displacement noise of the motion parallel to the reflective surface is also canceled within each of the brackets (due to the law of reflection); while displacement noise of the motion normal to the reflective surface is canceled by subtracting the two brackets. In a complete DFI scheme, e.g., the one constructed in Ref. [8], each optical component should have multiple links to other elements, such that both displacement and timing noise are canceled.

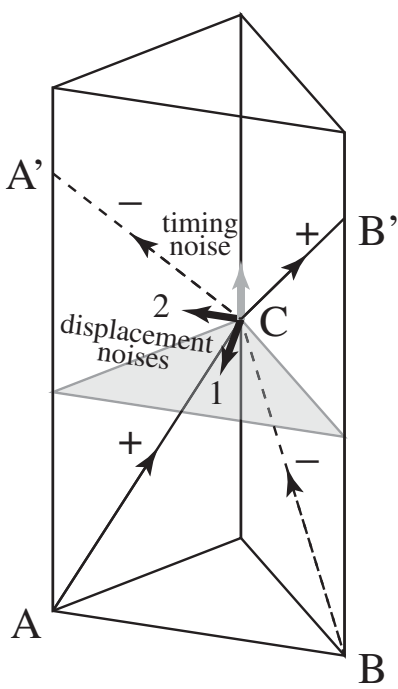

FIG. 2. 3-D space-time $(2+1)$ diagram illustrating cancellation of both timing and displacement noise.

\section{OVERVIEW OF DOUBLE-OCTAHEDRON CONFIGURATIONS}

In this section, we discuss briefly some common features of a class of schemes based on the single- or the doubleoctahedron geometry. We will focus on the doubleoctahedron configuration, but the argument with the single octahedron can be made as well by taking one of the octahedrons in the double octahedron.

\section{A. Geometry}

In Fig. 3, we construct a double-octahedron geometry, $A_{a}-C_{1} D_{1} C_{2} D_{2}-B_{a}$ in solid lines and $A_{b}-C_{1} D_{1} C_{2} D_{2}-B_{b}$ in

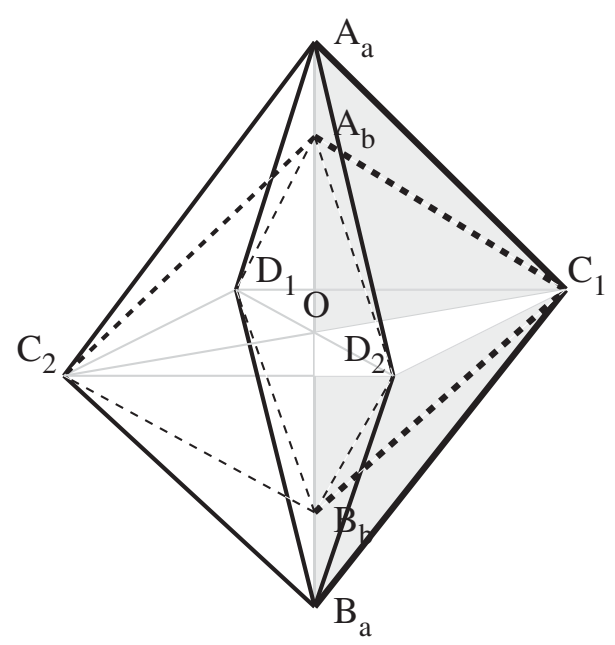

FIG. 3. Double-octahedron geometry, on which all configurations discussed in this paper are based. Here $C_{1} D_{1} C_{2} D_{2}$ is a square, with $\left|O C_{1}\right|=l, A_{a} O \perp C_{1} D_{1} C_{2} D_{2},\left|A_{a} O\right|=\left|B_{a} O\right|$, $\left|A_{b} O\right|=\left|B_{b} O\right|$. We also denote $\alpha_{a} \equiv \angle A_{a} C_{1} O$ and $\alpha_{b} \equiv$ $\angle A_{b} C_{1} O$. 
dashed lines, on which all schemes in this paper will be based. We shall call $A_{a, b}$ and $B_{a, b}$ apex vertices, while $C_{1,2}$ and $D_{1,2}$ base vertices. Note that these octahedrons are in general not regular. In our schemes, we only send light along the edges of the octahedrons (i.e., dashed and solid lines, but not gray lines in Fig. 3). Timing channels can then be grouped into four sections, i.e., those involving only one of the base vertices, $C_{1}, D_{1}, C_{2}$, or $D_{2}$, respectively. In the section involving $C_{1}$ (highlighted in Fig. 3 by a shaded triangle), for example, we have 8 timing channels $A_{a} C_{1}, C_{1} A_{a}, B_{a} C_{1}, C_{1} B_{a}, A_{b} C_{1}, C_{1} A_{b}, B_{b} C_{1}$, and $C_{1} B_{b}$ (highlighted in Fig. 3 by thicker lines). By making substitutions $C_{1} \rightarrow D_{1}, C_{1} \rightarrow C_{2}$, and $C_{1} \rightarrow D_{2}$, we obtain corresponding timing channels in all other sections. We only consider schemes in which corresponding timing channels in the $C_{1}, D_{1}, C_{2}$, and $D_{2}$ sections are combined with a +-+- fashion, as in Ref. [8]. For example, in the total combination $\tau$, the time delays $\tau_{C_{1} B_{b}}, \tau_{D_{1} B_{b}}, \tau_{C_{2} B_{b}}$, and $\tau_{D_{2} B_{b}}$ can only appear in the following way (in the frequency domain):

$$
\begin{aligned}
\tilde{\tau}= & \mathcal{F}(\Omega)\left[\tilde{\tau}_{C_{1} B_{b}}-\tilde{\tau}_{D_{1} B_{b}}+\tilde{\tau}_{C_{2} B_{b}}-\tilde{\tau}_{D_{2} B_{b}}\right] \\
& + \text { other channels, }
\end{aligned}
$$

where $\mathcal{F}(\Omega)$ is the frequency-dependent linear coefficient, which indicates the way this particular section is included in the total timing combination. Because of the symmetry built into our geometry, all such combinations are automatically free from timing and displacement noise at the apex vertices $\left(A_{a}, B_{a}, A_{b}\right.$, and $\left.B_{b}\right)$. We are only left to check noise-free conditions involving the base vertices $\left(C_{1}, D_{1}, C_{2}\right.$, and $\left.D_{2}\right)$, and to evaluate $\mathrm{GW}$ sensitivity.

\section{B. Shot noise in laser interferometry}

When a scheme based on measuring light travel time is implemented by interferometry, the shot-noise-limited sensitivity not only depends on the laser power, but also on exactly how the measurement is implemented. For example, suppose that in a scheme, we demand that $\tau_{A_{a} C_{1}}$ and $\tau_{C_{1} B_{a}}$ are measured immediately following each other; then there are two ways of realizing this using interferometry: (i) sending a beam from $A_{a}$ to $C_{1}$, comparing its phase with a local oscillator at $C_{1}$, also sending a beam from $C_{1}$ (phase-locked with the above-mentioned local oscillator at the level of shot noise), comparing its phase with a local oscillator at $B_{a}$, upon arrival; or (ii) putting a mirror at $C_{1}$, which reflects the light beam from $A_{a}$ towards $B_{a}$, eliminating the need for the local oscillator and the local laser at $C_{1}$. Obviously, in the ideal situation (e.g., in absence of optical losses), scheme (ii) has less shot noise.

In fact, in all the schemes we shall consider in this paper, we always implement them by sending light from the apex vertices and receiving them at their antipodal apex vertices - with base vertices only as relays. Beams propagating in different sections or different octahedrons also have independent shot noise. In this way, the equivalent shot noise in $\tau_{A_{a} C B_{a}}, \tau_{B_{a} C A_{a}}, \tau_{A_{b} C B_{b}}$, and $\tau_{B_{b} C A_{b}}$ are all independent, with

$$
\begin{aligned}
& S_{\tau_{A_{a} C B_{a}}}=S_{\tau_{B_{a} C A_{a}}}=\frac{\hbar}{2 I_{a} \omega_{0}}, \\
& S_{\tau_{A_{b} C B_{b}}}=S_{\tau_{B_{b} C A_{b}}}=\frac{\hbar}{2 I_{b} \omega_{0}},
\end{aligned}
$$

where $I_{a, b}$ are powers of light beams traveling along the edges of the $a$ - and $b$-octahedrons, and $\omega_{0}$ is the light angular frequency.

\section{DFI WITH TIME DELAY}

\section{A. Single octahedron}

First, we attempt to make the simplest modification to the single-octahedron configuration to introduce time delays, namely, by assuming a time delay each time the light reflects off mirror $C_{1,2}$ and $D_{1,2}$ - due to the existence of a rigid "time-delay cell," which imposes the same time delay $T_{d}$ and delay noise on beams entering the cell at the same time from opposite directions. A section of this configuration is illustrated in the panel (i) of Fig. 4. It is straightforward to deduce that

$$
\begin{gathered}
\tau_{A C_{A}}(t)=[y(t)-z(t)] / \sqrt{2}, \\
\tau_{C_{A} C_{B}}(t) \equiv 0, \\
\tau_{C_{B} B}(t)=[y(t-L)+z(t-L)] / \sqrt{2} .
\end{gathered}
$$

Note that the argument $t$ in each of the $\tau$ 's represent the ending time of the link. We then have

$$
\begin{aligned}
\tau_{A C_{A} C_{B} B}(t)= & \tau_{C_{B} B}(t)+\tau_{C_{A} C_{B}}(t-L)+\tau_{A C_{A}}\left(t-L-T_{d}\right) \\
= & {\left[y(t-L)+z(t-L)+y\left(t-L-T_{d}\right)\right.} \\
& \left.-z\left(t-L-T_{d}\right)\right] / \sqrt{2} .
\end{aligned}
$$

Similar steps get

$$
\begin{aligned}
\tau_{B C_{B} C_{A} A}(t)= & \tau_{C_{A} A}(t)+\tau_{C_{B} C_{A}}(t-L)+\tau_{B C_{B}}\left(t-L-T_{d}\right) \\
= & {\left[y(t-L)-z(t-L)+y\left(t-L-T_{d}\right)\right.} \\
& \left.+z\left(t-L-T_{d}\right)\right] / \sqrt{2} .
\end{aligned}
$$

Making a subtraction, one obtains

$$
\tau_{A C_{A} C_{B} B}(t)-\tau_{B C_{B} C_{A} A}(t)=\sqrt{2}\left[z(t-L)-z\left(t-L-T_{d}\right)\right] .
$$

When $T_{d} \neq 0$, this displacement noise is not canceled.

In this scheme, we also see that fluctuations in the difference of time delays imposed by the delay cell onto the two counter-propagating beams also can cause noise. This can be circumvented completely by replacing the monolithic time-delay cell to a pair of cells shown in the 
(i)
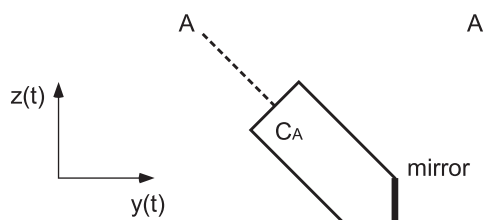

(ii)

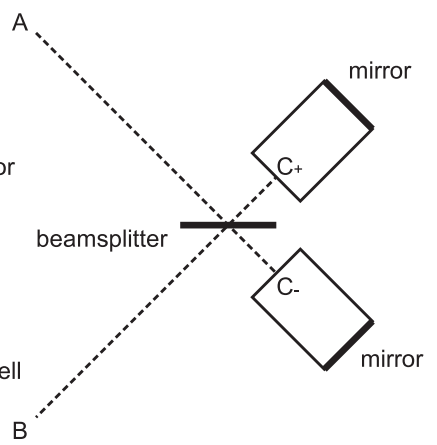

(iii)

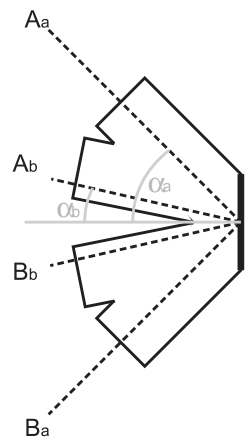

(iv)

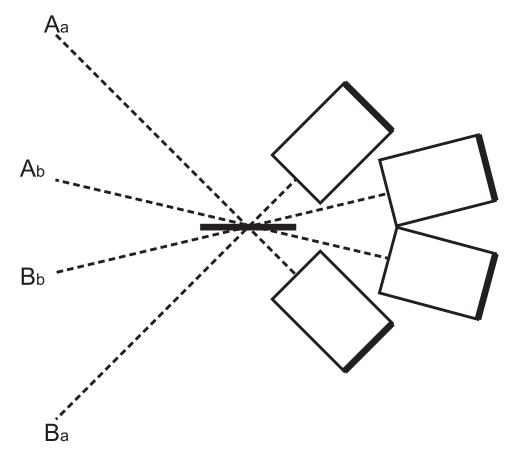

FIG. 4. (i), (ii): a section of the time-delayed single-octahedron scheme discussed in Sec. IVA. (iii), (iv): a section of the timedelayed double-octahedron schemes discussed in Secs. IV B and V C.

panel (ii) of Fig. 4 [11]. After being split, two beams coming from different directions experience the same time delay, which is the average of two time delays. However, the $z$ motion of the device, which here corresponds to the motion of the beam splitter, cannot be removed. In Ref. [11], this $z$ motion is not considered appropriately in some cases; a black dotted curve in its Fig. 7 and black curves in its Figs. 9 and 12 should be modified. While the motion of the beam splitter between the time-delay devices is taken into account, it is not in the case a monolithic time-delay device is assumed.

\section{B. Double-octahedron with delay-noise cancellation}

It is possible to combine two of the above time-delayed octahedron configurations, and cancel their sensitivities to $z$ motions. This can be realized by a double-octahedron configuration [11] shown in Fig. 3. Time-delay cells are implemented in the way shown in the panel either (iii) or (iv) of Fig. 4. For each of the octahedrons, $a$ and $b$, we have, by generalizing Eqs. (9)-(14),

$$
\begin{aligned}
\tau_{a}(t)= & \tau_{A_{a} C B_{a}}(t)-\tau_{B_{a} C A_{a}}(t) \\
= & 2 \sin \alpha_{a}\left[z\left(t-L / \cos \alpha_{a}\right)\right. \\
& \left.-z\left(t-L / \cos \alpha_{a}-T_{d}\right)\right], \\
\tau_{b}(t)= & \tau_{A_{b} C B_{b}}(t)-\tau_{B_{b} C A_{b}}(t) \\
= & 2 \sin \alpha_{b}\left[z\left(t-L / \cos \alpha_{b}\right)\right. \\
& \left.-z\left(t-L / \cos \alpha_{b}-T_{d}\right)\right] .
\end{aligned}
$$

As a consequence, we demand the following combination (within this section) in order to cancel displacement noise:

$$
\begin{aligned}
\tau_{\text {section }}= & \sin \alpha_{b} \tau_{a}\left(t-L / \cos \alpha_{b}\right) \\
& -\sin \alpha_{a} \tau_{b}\left(t-L / \cos \alpha_{a}\right) .
\end{aligned}
$$

In Fig. 5, we plot $\sqrt{S_{h}^{\text {shot }}}$ of this DFI configuration, assuming $L=3 \mathrm{~km}, T_{d}=10 \mathrm{~ms}, \alpha_{a}=\pi / 4$, and $\alpha_{b}=$ $\pi / 6$. We have set an input power of $100 \mathrm{MW}$, which can be realized by a $100 \mathrm{~W}$ input laser with signal and power recycling gains of 1000 each. Such optical parameters are, although not at the same time, respectively realizable with the current technology $[14,15]$. The resulting noise spectrum is consistent with $\alpha=2$ [cf. Eq. (1)], and thus does not offer any improvement over existing DFI schemes. (In calculating $S_{h}$, we have assumed the GW to propagate along $O A$ direction.) In the same figure, the design sensitivity of LIGO-I has been plotted. Even though the DFI

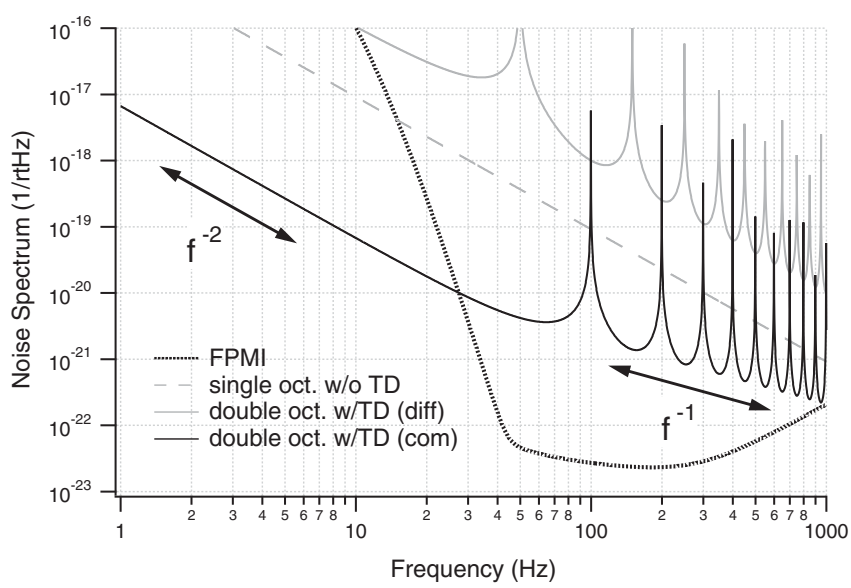

FIG. 5. DFI shot-noise spectrum compared with the total noise spectrum of a conventional GW detector. The arm length of each detector is set to $3 \mathrm{~km}$. The spectrum of a single octahedron without time delay (gray dashed line) follows a $f^{-2}$ power law in the observation band $\left(\Omega<\Omega_{\text {peak }}\right)$. The spectrum of a timedelayed double octahedron with delay-noise cancellation (indicated as diff. shown in a gray solid curve) cannot be better than that of the single octahedron. The spectrum of a time-delayed double octahedron without delay-noise cancellation (indicated as com, shown in black solid curve) follows the three-part power law (2), and thus has a better sensitivity than the single octahedron. However, delay noise would limit the sensitivity at almost all the frequencies. Here the time delay is set to $10 \mathrm{~ms}$. 
noise spectra are lower than LIGO-I for frequencies below $\sim 10-20 \mathrm{~Hz}$, they do not offer meaningful GW sensitivity.

\section{INCOMPATIBILITY BETWEEN TIME-DELAY- NOISE CANCELLATION AND IMPROVEMENT IN SENSITIVITY}

\section{A. General discussion}

In Sec. IV, we tried a few example configurations with the octahedron structure to implement time delay into DFI but could not find a successful noise-free solution to make $\alpha=1$. In this section, we argue that this is a general fact, by making a systematic discussion, treating the interferometer scheme as an array of test masses, or nodes, with light traveling between these nodes, and light travel times measured-as done in Ref. [7]. Suppose we focus on a node $C$ in the array with time-delay devices, and $C$ relays light from $A_{1}$ to $B_{1}, A_{2}$ to $B_{2}, \ldots, A_{n}$ to $B_{n}$, each with a time delay of $T_{A_{j} C B_{j}}+\delta_{A_{j} C B_{j}}(t), j=1, \ldots, n$. We also suppose $C$ to relay light backwards from $B_{1}$ to $A_{1}$, etc., with time delay $T_{A_{j} C B_{j}}=T_{B_{j} C A_{j}}$. Here $T_{A_{j} C B_{j}}$ is the nominal delay time, $\delta_{A_{j} C B_{j}}(t)$ is the delay noise at time $t$. As in Refs. [6,7], we use the TT gauge and with $t$ we denote the coordinate time (upon the light's exit from the delay device), which coincides with the proper time of the devices with error $O\left[(v / c)^{2}\right]$. The timing signal $\tau_{A_{j} C B_{j}}(t)$ (with $t$ time of receipt) is then (up to linear order in GW and various types of noise)

$$
\begin{aligned}
\tau_{A_{j} C B_{j}}(t)= & \tau_{\mathrm{GW}}\left[C \rightarrow B_{j} ; t\right]+\tau_{\mathrm{GW}}\left[A_{j} \rightarrow C ; t-L_{C B_{j}}-T_{A_{j} C B_{j}}\right]+\tau_{B_{j}}(t)-\tau_{A_{j}}\left(t-L_{C B_{j}}-T_{A_{j} C B_{j}}-L_{A_{j} C}\right) \\
& +\delta_{A_{j} C B_{j}}\left(t-L_{C B_{j}}\right)+\mathbf{n}_{C B_{j}} \cdot\left[\mathbf{x}_{B_{j}}(t)-\mathbf{x}_{C_{j}}\left(t-L_{C B_{j}}\right)\right]+\mathbf{n}_{A_{j} C} \cdot\left[\mathbf{x}_{C_{j}}\left(t-L_{C B_{j}}-T_{A_{j} C B_{j}}\right)\right. \\
& \left.-\mathbf{x}_{A_{j}}\left(t-L_{C B_{j}}-T_{A_{j} C B_{j}}-L_{A_{j} C}\right)\right] .
\end{aligned}
$$

On the other hand, given for the same array, but without time-delay devices, we could construct the following timing channels, to prepare for a " $*$ " configuration:

$$
\begin{aligned}
\tau_{C B_{j}}^{*}(t)= & \tau_{\mathrm{GW}}\left[C \rightarrow B_{j} ; t\right]+\tau_{B_{j}}(t)-\tau_{C}\left(t-L_{C B_{j}}\right)+\mathbf{n}_{C B_{j}} \cdot\left[\mathbf{x}_{B_{j}}(t)-\mathbf{x}_{C_{j}}\left(t-L_{C B_{j}}\right)\right], \\
\tau_{A_{j} C}^{*}\left(t-L_{C B_{j}}-T_{A_{j} C B_{j}}\right)= & \tau_{\mathrm{GW}}\left[A_{j} \rightarrow C ; t-L_{C B_{j}}-T_{A_{j} C B_{j}}\right]+\tau_{C}\left(t-L_{C B_{j}}-T_{A_{j} C B_{j}}\right)-\tau_{A_{j}}\left(t-L_{C B_{j}}-T_{A_{j} C B_{j}}-L_{A_{j} C}\right) \\
& +\mathbf{n}_{C B_{j}} \cdot\left[\mathbf{x}_{B_{j}}(t)-\mathbf{x}_{C_{j}}\left(t-L_{C B_{j}}\right)\right]+\mathbf{n}_{A_{j} C} \cdot\left[\mathbf{x}_{C_{j}}\left(t-L_{C B_{j}}-T_{A_{j} C B_{j}}\right)\right. \\
& \left.-\mathbf{x}_{A_{j}}\left(t-L_{C B_{j}}-T_{A_{j} C B_{j}}-L_{A_{j} C}\right)\right] .
\end{aligned}
$$

We therefore have

$$
\begin{aligned}
& \tau_{A_{j} C B_{j}}\left(t+L_{C B_{j}}\right)-\left[\tau_{C B_{j}}^{*}\left(t+L_{C B_{j}}\right)+\tau_{A_{j} C}^{*}\left(t-T_{A_{j} C B_{j}}\right)\right]=\delta_{A_{j} C B_{j}}(t)+\tau_{C}\left(t-T_{A_{j} C B_{j}}\right)-\tau_{C}(t), \\
& \tau_{B_{j} C A_{j}}\left(t+L_{C A_{j}}\right)-\left[\tau_{C A_{j}}^{*}\left(t+L_{C A_{j}}\right)+\tau_{B_{j} C}^{*}\left(t-T_{B_{j} C A_{j}}\right)\right]=\delta_{B_{j} C A_{j}}(t)+\tau_{C}\left(t-T_{B_{j} C A_{j}}\right)-\tau_{C}(t) .
\end{aligned}
$$

Equations (21) and (22) therefore establish a correspondence between each time-delay configuration and a * configuration; the corresponding configurations have the same GW response, same amount of displacement noise, while their timing noise only differ due to terms on the right-hand side. At the same time, the $\delta$ terms on the righthand sides of Eqs. (21) and (22) are exactly the time-delaynoise contributions from our time-delay links, while the $\tau_{C}$ terms are exactly the $C$-timing-noise contributions in the * configuration.

Now, in order to cancel delay noise, we must assume some of the delay-noise contributions $\delta_{A_{j} C B_{j}}$ and $\delta_{B_{j} C A_{j}}$ are related - otherwise we cannot hope to have vanishing total delay noise. In addition, assuming $A_{j}$ 's and $B_{j}$ 's to be different from each other, it is only reasonable to assume that $\delta_{B_{j} C A_{j}}(t)=\delta_{A_{j} C B_{j}}(t)$. It is then only possible to construct time-delay-noise-free configurations by combining Eqs. (21) and (22) with an opposite sign (plus some com- mon time-delay manipulations). However, having assumed $T_{B_{j} C A_{j}}=T_{A_{j} C B_{j}}$, we infer that the $*$ configuration, realizable without time delay, is also free from timing noise. As a consequence, any DFI configuration with time-delay devices and delay-noise cancellation can be realized by a DFI configuration without time-delay devices at all.

However, since a DFI configuration without time-delay devices satisfy Eq. (1) and have $\alpha=2$ at best (realized in 3-D configurations) - which implies that we cannot hope to achieve the three-part power law (2), if we insist on the cancellation of delay noise.

\section{B. Discussions with 3-D space-time diagrams}

The situation of the single octahedron with a single-path time delay that we showed in Sec. IVA is illustrated in the left panel of Fig. 6. As seen there, our combination senses the positions of $C$ and $C^{\prime}$ (i.e., the object $C$ after a long time delay) along the $A B$ direction (shown in the figure by thick 

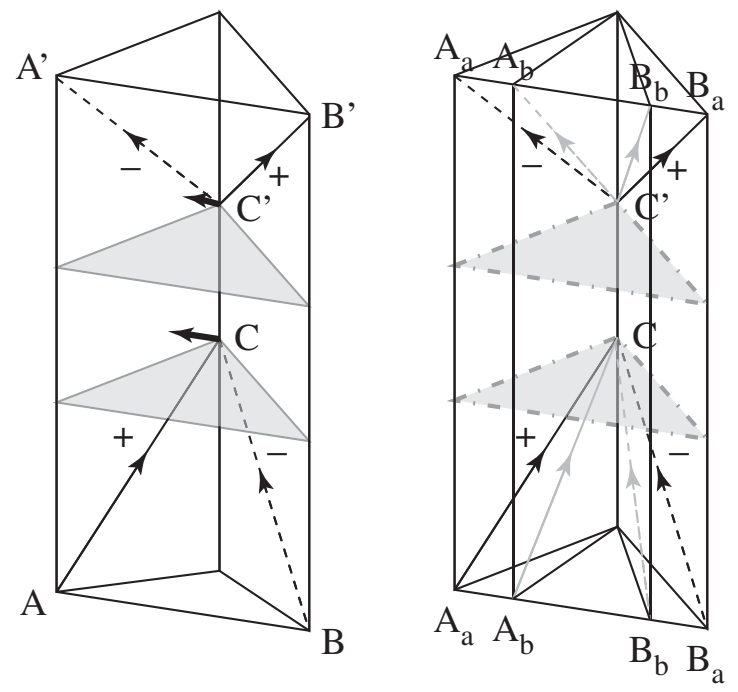

FIG. 6. Time-delay configurations that either fail to cancel displacement noise or fail to improve sensitivity. Left panel: a naive extension of the 3-D octahedron of Ref. [8]; motion of $C$ along reflective surface is not canceled, due to time delay. Right panel: 3-D configuration with time-delay-noise cancellation, the entire configuration can be cut in two, along the two shaded triangles, each without time-delay devices, and free from displacement noise and timing noise, and with $\alpha=2$.

solid arrows), and makes a subtraction. However, since these positions can be different, we do not cancel displacement noise arising from motion of $C$ along the $A B$ direction. From the space-time diagram, it does not seem possible to cancel displacement noise of both $C$ and $C^{\prime}$, in both directions, with a single-octahedron configuration.

The right panel of Fig. 6 shows the space-time diagram for the double-octahedron configuration that we showed in Sec. IV B. On the one hand, it is easy to check that this configuration can cancel delay noise because the links leading to $C$ from $A_{a}$ and $B_{a}$ are subtracted from each other (so are links emerging from $C^{\prime}$ to $A_{a}^{\prime}$ and $B_{a}^{\prime}$, etc.). On the other hand, looking at the right panel of Fig. 6, it is easy to convince oneself that the space-time diagram of this double-octahedron scheme can be cut into two disconnected pieces separated by $T_{d}$ in time, each free from displacement and timing noise. In other words, our scheme can be simulated by running two time-delay-free DFI devices successively, separated by $T_{d}$ in time. As a consequence, we also expect $\alpha=2$ for this scheme.

\section{Double-octahedron without delay-noise cancellation}

In order to reach $\alpha=1$, we must modify our schemes and make them sensitive to delay noise. The building block of such schemes in a section of a single octahedron (i) in Fig. 4 is

$$
\tau_{A C B}(t)+\tau_{B C A}(t) .
$$

We show the 3-D space-time diagram of this part of the scheme in the left panel of Fig. 7. We note that delay noise, as well as the $y$ motion of $C$ (i.e., normal to the reflective surface) are not canceled (since we add up the two timing signals, sensitivity to $z$ cancels). We now go to the doubleoctahedron configuration (iii) in Fig. 4, and denote by $l$ the distance between the center of gravity and the center of the cell. The remaining $y$ sensitivity is

$$
\begin{aligned}
\tau_{a}(t)= & \tau_{A_{a} C B_{a}}(t)+\tau_{B_{a} C A_{a}}(t) \\
= & 2 \cos \alpha_{a}\left[y\left(t-L / \cos \alpha_{a}\right)\right. \\
& \left.+y\left(t-L / \cos \alpha_{a}-T_{d}\right)\right], \\
\tau_{b}(t)= & \tau_{A_{b} C B_{b}}(t)+\tau_{B_{b} C A_{b}}(t) \\
= & 2 \cos \alpha_{b}\left[y\left(t-L / \cos \alpha_{b}\right)\right. \\
& \left.+y\left(t-L / \cos \alpha_{b}-T_{d}\right)\right] .
\end{aligned}
$$

So the required combination in this section to cancel $y$ is

$$
\begin{aligned}
\tau_{\text {section }}= & \cos \alpha_{b} \tau_{a}\left(t-L / \cos \alpha_{b}\right) \\
& -\cos \alpha_{a} \tau_{b}\left(t-L / \cos \alpha_{a}\right) .
\end{aligned}
$$

Note that cancellation of $y$ is not compatible with cancellation of delay noise [which requires Eq. (26) without prefactors of $\cos \alpha_{a}$ and $\cos \alpha_{b}$ ], and $\tau$ is still sensitive to delay noise.

In Fig. 5, we plot the shot-noise spectrum of this configuration, compared with DFI without time delay, and double octahedron with delay-noise cancellation. The parameters are the same as what we showed in Sec. IV B. This configuration follows the three-part power law (2),
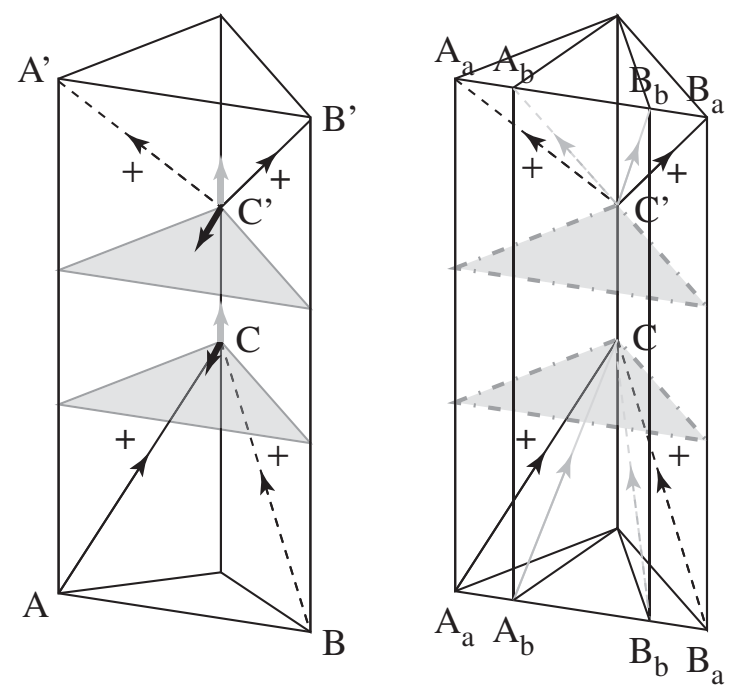

FIG. 7. Time-delay configurations that do not cancel delay noise. Left panel: building block from a single octahedron. Right panel: full double-octahedron DFI configuration with $\alpha=$ 1; unlike in the right panel of Fig. 6, cutting this scheme along the shaded triangles does not give two DFI schemes (because timing-noise contributions at $C$ and $C^{\prime}$ are not canceled). 
and thus has a much better shot-noise-limited sensitivity than previous DFI configurations. However, we should note that delay noise simply ignored here is not negligible but instead very significant, as is shown in the appendix. In addition, even if free from delay noise, this configuration still does not provide a very interesting astrophysical sensitivity, despite its complexity.

\section{CONCLUSION AND DISCUSSION}

In this paper, we investigated the possibility of using time-delay devices to improve the sensitivity of displacement-noise-free $\mathrm{GW}$ detectors.

A three-dimensional DFI with time delays of $\mathcal{T}_{D}$ can provide a three-part power law (2), which has dramatically better shot-noise-limited sensitivity compared to the twopart power law (1) of DFI schemes without time-delay devices. However, as we have argued in Sec. V, such schemes must not cancel fluctuations in the artificial time delays. In order for delay noise not to exceed shot noise, we must put an extremely high requirement on our time-delay device: $\sqrt{S_{\delta}^{-}}<\mathcal{L} \sqrt{S_{h}^{\text {shot }}} / c$.

In the appendix, we consider time-delay fluctuations in a particular type of time-delay devices based on EIT. Although EIT time delay has a wide application in other fields, our requirement in time-delay DFI schemes seems extremely challenging for such devices.

\section{ACKNOWLEDGMENTS}

We wish to thank Seiji Kawamura, Archana Pai, and Atsushi Nishizawa for valuable discussions. Research of K. S. and Y.C. are supported by the Sofja Kovalevskaya Program of the Alexander von Humboldt Foundation (funded by the German Federal Ministry of Education and Research). K. G. is supported by the U.S. National Science Foundation under Cooperative Agreement No. PHY-0107417 and PHY-0457264.

\section{APPENDIX: THERMAL NOISE OF ATOMS IN AN EIT MEDIUM}

In this appendix, we estimate a noise level of an EIT medium as a time-delay device. A time-delay device with EIT is realized by a cell filled by vapor atoms and pump field injected to the cell in addition to the probe light that measures gravitational waves. While the cell does not transmit the probe light without the pump field, a transition of the atomic state due to the interaction with the pump field makes the cell transparent to the light at a particular frequency. The transition makes a refractive index of the cell rapidly vary and slows group velocity of the light, which results in the time-delay effect $[13,14]$. Fluctuation of the time delay, or EIT noise, comes from thermal motion of atoms. The probe light will see different numbers of atoms at different times. The time delay is proportional to the number of atoms that interact with the probe light, so the number fluctuation makes the time-delay fluctuation. Wiener-Khintchine's theorem reads a power spectral density of fluctuation $x(t)$ is

$$
S_{x}(\Omega)=\lim _{T \rightarrow \infty} \frac{1}{2 \pi T} \iint_{T / 2}^{T / 2}\left\langle x(t) x\left(t^{\prime}\right)\right\rangle e^{-i \Omega\left(t-t^{\prime}\right)} d t d t^{\prime} .
$$

In the case where we have a single beam in an EIT medium, the self-correlation function will be written as

$$
\begin{aligned}
C\left(t, t^{\prime}\right)= & \left\langle\delta \tau(t) \delta \tau\left(t^{\prime}\right)\right\rangle \\
= & \frac{1}{v_{g}^{2} \rho^{2}} \iint_{0}^{L e} d \ell d \ell^{\prime} \iiint \int d x d x^{\prime} d y d y^{\prime} \\
& \times A(x, y) A^{\prime}\left(x^{\prime}, y^{\prime}\right) \times\left\langle\delta \rho(t ; \ell) \delta \rho\left(t^{\prime} ; \ell^{\prime}\right)\right\rangle .
\end{aligned}
$$

Here, $L_{e}$ is a length of the medium, $v_{g}$ is the averaged group velocity of the light, $\rho$ is density of atoms, and $A$ is the beam profile.

In the case where we have two counter-propagating beams to one EIT medium, where only a differential mode of the fluctuations matters as we will subtract the outputs to obtain the signal of DFI, it is not only the selfcorrelation function but also the cross-correlation function between two beams that should be taken into account. The light starting the left side of the medium at $t=t_{0}$ probes the fluctuation in a sliced region $\delta V(\ell)$ at $t=t_{0}+\ell / v_{g}$, and the light starting the right side of the medium at $t=t_{0}$ probes the fluctuation in the same sliced region $\delta V(\ell)$ at $t=t_{0}+\left(L_{e}-\ell\right) / v_{g}$. The autocorrelation function will be rewritten into

$$
\begin{aligned}
C_{d}\left(t, t^{\prime}\right)= & \left\langle\delta \tau_{d}(t) \delta \tau_{d}\left(t^{\prime}\right)\right\rangle \\
= & \frac{1}{v_{g}^{2} \rho^{2}} \iint_{0}^{L e} d \ell d \ell^{\prime} \iiint \int d x d x^{\prime} d y d y^{\prime} \\
& \times A(x, y) A^{\prime}\left(x^{\prime}, y^{\prime}\right)\left\langle\left\{\delta \rho\left(\frac{\ell}{v_{g}}+t ; \ell\right)\right.\right. \\
& \left.-\delta \rho\left(\frac{L_{e}-\ell}{v_{g}}+t ; \ell\right)\right\}\left\{\delta \rho\left(\frac{\ell^{\prime}}{v_{g}}+t^{\prime} ; \ell^{\prime}\right)\right. \\
& \left.\left.-\delta \rho\left(\frac{L_{e}-\ell^{\prime}}{v_{g}}+t^{\prime} ; \ell^{\prime}\right)\right\}\right\rangle .
\end{aligned}
$$

After some calculations, we have

$$
S_{\tau}^{\text {one }}(\Omega) \simeq \frac{\tau_{\mathrm{TD}}^{2}}{N} \tau_{M} e^{-\Omega \tau_{M}}
$$

for the single beam, and

$$
S_{\tau}^{\mathrm{diff}}(\Omega) \simeq \frac{\tau_{\mathrm{TD}}^{2}}{N} \tau_{M} e^{-\Omega \tau_{M}}\left(1-\frac{v_{T}}{L_{e} \Omega} \sin \left[\frac{L_{e} \Omega}{v_{T}}\right]\right)
$$

for the counter-propagating beams. Here $\tau_{\mathrm{TD}}=L_{e} / v_{g}$ is the time delay, $N$ is the effective number of atoms in the Gaussian beam, and $\tau_{M}=\sqrt{2} w / v_{T}$ is averaged time that an atom stays in the beam according to the Maxwell 
distribution ( $w$ is a beam radius and $v_{T}$ is the averaged speed of the atoms). Note that only the calculation with a single beam is meaningful since subtraction of the outputs of the counter-propagating beams results in the cancellation of the time-delay effect. Equation (A5) rather represents the existence of differential delay noise even if we do the subtraction with a monolithic cell.

Let us put the time delay of $\tau_{\mathrm{TD}}=0.01 \mathrm{~s}$ into Eq. (A4). Typical parameters $L_{e}=10 \mathrm{~cm}, w=1 \mathrm{~cm}, \quad v_{T}=$ $300 \mathrm{~m} / \mathrm{s}$, and $N=10^{14}$, which correspond to the atom density of $10^{-12} \mathrm{~cm}^{-3}$, give us the EIT-noise level at $50 \mathrm{~Hz}$, for example, as $\sqrt{S_{\tau}^{\text {one }}} \sim 7 \times 10^{-12} \mathrm{~s} / \sqrt{\mathrm{Hz}}$. In displacement, this is $\sim 2 \mathrm{~mm} / \sqrt{\mathrm{Hz}}$, which is much larger than other noise in gravitational wave detectors. The noise level decreases exponentially from $f \sim 1 /\left(2 \pi \tau_{M}\right) \sim 3 \mathrm{kHz}$. One could make this decay start from frequencies as low as $5 \mathrm{~Hz}$, but it requires the temperature as low as $0.8 \mathrm{mK}$ or the beam radius as large as $6 \mathrm{~m}$, which would be challenging in practice.
[1] D. Shoemaker (LIGO Scientific Collaboration), Nucl. Instrum. Methods Phys. Res., Sect. A 517, 154 (2004).

[2] L. Di Fiore (VIRGO collaboration), Classical Quantum Gravity 19, 1421 (2002).

[3] B. Willke et al., Classical Quantum Gravity 19, 1377 (2002).

[4] M. Ando (TAMA collaboration), Phys. Rev. Lett. 86, 3950 (2001).

[5] P. Bender et al., LISA Pre-Phase A Report, 1998, 2nd edition.

[6] S. Kawamura and Y. Chen, Phys. Rev. Lett. 93, 211103 (2004).

[7] Y. Chen and S. Kawamura, Phys. Rev. Lett. 96, 231102 (2006).

[8] Y. Chen et al., Phys. Rev. Lett. 97, 151103 (2006).
[9] S. Sato et al., Phys. Rev. Lett. 98, 141101 (2007).

[10] M. Rakhmanov, arXiv:gr-qc/0406009.

[11] K. Somiya et al., J. Phys.: Conf. Ser., 66, 012053 (2007). Note that displacement noise of the motion orthogonal to the beam is partially not taken into account in this paper (see Sec. IVA).

[12] A. Nishizawa et al., Proceedings of the JGRG meeting, Niigata, 2006.

[13] S. E. Harris, J. E. Field, and A. Kasapi, Phys. Rev. A 46, R29 (1992).

[14] D. Budker, D.F. Kimball, S. M. Rochester, and V. V. Yashchuk, Phys. Rev. Lett. 83, 1767 (1999).

[15] B. Willke, Proceedings of the LSC meeting, Baton Rouge, 2007. 